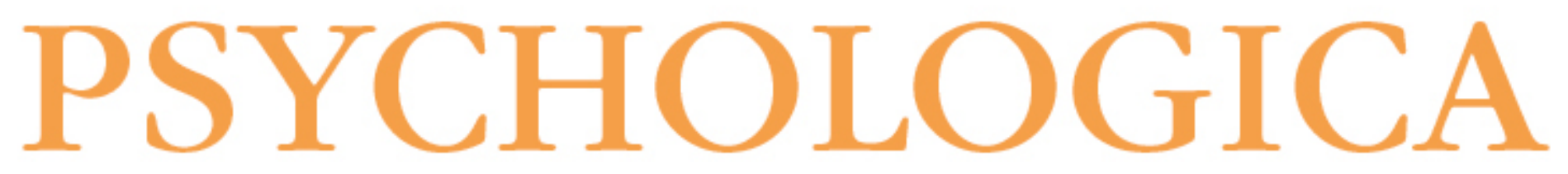

\title{
Promoção da saúde na doença oncológica: intervenção de redução do stress baseada no mindfulness
}

Autor(es): $\quad$ Teixeira, Ricardo; Pereira, M. Graça

Publicado por: Imprensa da Universidade de Coimbra

URL

persistente: URI:http://hdl.handle.net/10316.2/5419

DOI: $\quad$ DOI:http://dx.doi.org/10.14195/1647-8606_50_13

Accessed : $\quad$ 26-Apr-2023 15:04:23

A navegação consulta e descarregamento dos títulos inseridos nas Bibliotecas Digitais UC Digitalis, UC Pombalina e UC Impactum, pressupõem a aceitação plena e sem reservas dos Termos e Condições de Uso destas Bibliotecas Digitais, disponíveis em https://digitalis.uc.pt/pt-pt/termos.

Conforme exposto nos referidos Termos e Condições de Uso, o descarregamento de títulos de acesso restrito requer uma licença válida de autorização devendo o utilizador aceder ao(s) documento(s) a partir de um endereço de IP da instituição detentora da supramencionada licença.

Ao utilizador é apenas permitido o descarregamento para uso pessoal, pelo que o emprego do(s) título(s) descarregado(s) para outro fim, designadamente comercial, carece de autorização do respetivo autor ou editor da obra.

Na medida em que todas as obras da UC Digitalis se encontram protegidas pelo Código do Direito de Autor e Direitos Conexos e demais legislação aplicável, toda a cópia, parcial ou total, deste documento, nos casos em que é legalmente admitida, deverá conter ou fazer-se acompanhar por este aviso. 


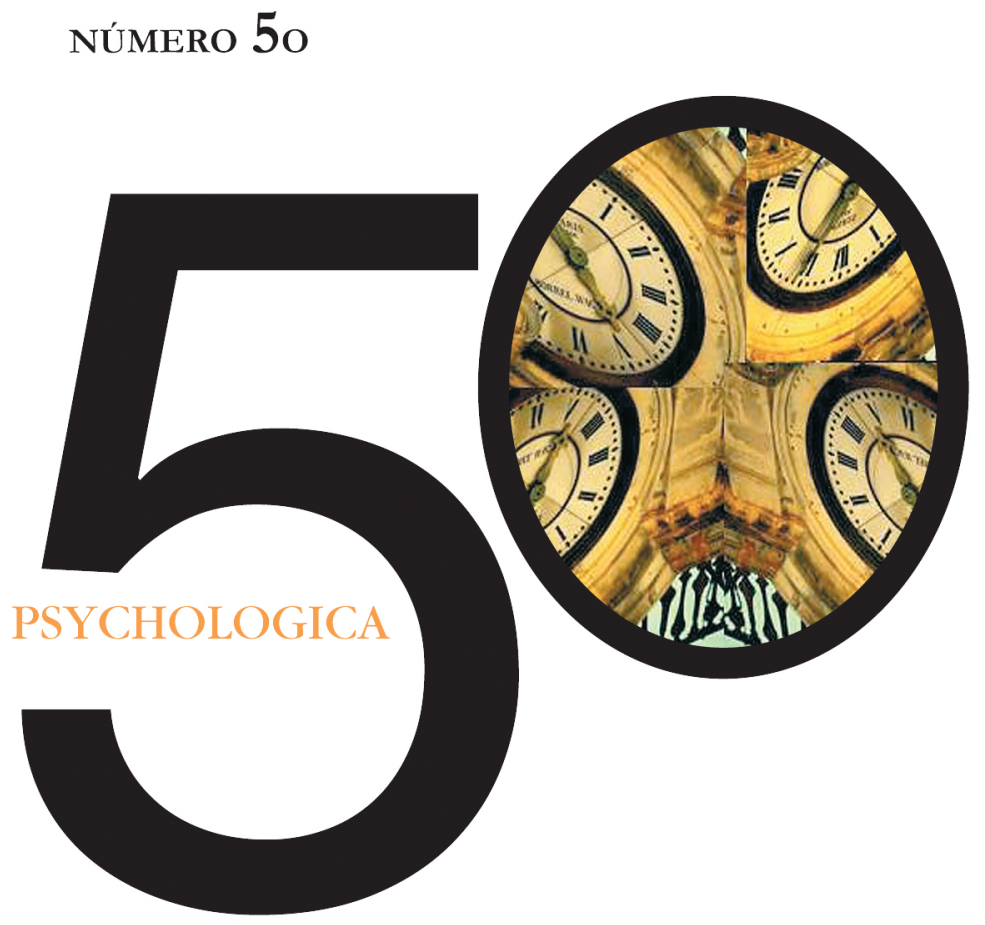

IMPRENSA DA UNIVERSIDADE DE COIMBRA

FACULDADE DE PSICOLOGIA E DE CIÊNCIAS DA EDUCAÇÃO DA UNIVERSIDADE DE COIMBRA 


\title{
Promoção da saúde na doença oncológica: Intervenção de redução do stress baseada no mindfulness
}

\author{
Ricardo Teixeira' \& M. Graça Pereira²
}

O objectivo deste artigo de revisão é (1) fornecer uma visão geral e uma discussão acerca da meditação mindfulness e sua aplicabilidade clínica em oncologia e (2) relatar detalhadamente avaliando criticamente a pesquisa existente e emergente sobre a meditação mindfulness como uma intervenção para doentes com cancro. Para além disso, pretende lançar uma hipótese manualizada de um programa de promoção da saúde e de redução do stress, baseado no mindfulness, explorando os seus efeitos em termos de variáveis biológicas e psicossociais. Estudos estrangeiros referem níveis significativos de mudança em itens psicossociais de avaliação, nos diferentes momentos de avaliação, pelo que a intervenção tem relativa sustentação teórica, embora a necessitar de refinamento empírico. Espera-se que a eventual aplicação da intervenção descrita neste artigo permita trabalhar os factores tidos como benéficos para um melhor ajustamento à doença oncológica, aumentando proactivamente a acção do indivíduo doente como interveniente informado do seu processo de adaptação progressiva à doença.

PALAVRAS-CHAVE: Mindfulness; Oncologia; Stress; Intervenção; Estudo teórico.

\section{Introdução}

O objectivo deste artigo é o de fazer uma pequena revisão acerca da aplicação dos programas de redução do stress baseados na meditação mindfulness em população oncológica. São exploradas as principais estratégias de promoção da saúde, e a sua incidência em termos de qualidade de vida. É feita uma revisão dos principais programas de intervenção em doentes oncológicos, e elaborado um enquadramento do programa de redução do stress baseado no mindfulness. É feita uma revisão conceptual e empírica do mindfulness, assim como uma breve apresentação da praticabilidade do programa. São ponderadas as possibilidades

\footnotetext{
1 Universidade do Minho (ricardojft@gmail.com) 2 Universidade do Minho (gracep@iep.uminho.pt)
} 
e contextos de implementação do programa em estudos com populações oncológicas em Portugal.

\section{Estratégias de promoção da saúde na doença oncológica}

As estratégias de promoção da saúde relacionam-se com os estilos de vida (escolhas individuais feitas num contexto social), e podem ter uma poderosa influência nos vectores da saúde. Um desses vectores relaciona-se com o grave flagelo pessoal, familiar e social, que abrange as doenças oncológicas (Gorin, 1998).

Para dar resposta de uma forma eficaz e correcta às reacções psicológicas que vivenciam os doentes oncológicos, é importante seguir uma matriz teórica que seja capaz de situar a variedade e inter-relação de todos os factores em que o doente está imerso. Desde o modelo biopsicossocial da saúde que se considera que, na abordagem de uma doença tão completa como o cancro, não intervêm apenas elementos biomédicos, sendo imprescindivel ter em conta os factores psicológicos e sociais relacionados com o surgimento e progressão do cancro. Deve ser considerada a multiplicidade de situações clínicas, em termos médico-psicológicos, à qual se deverão adequar técnicas psicoterapêuticas, incidindo todas elas no facto de que o indivíduo doente não quer estar doente e que sofre dos sintomas da doença (Barreto, Ferrero, \& Toledo, 1993). Assim, as estratégias devem basear-se em evidências científicas, derivadas da psico-oncologia, psicologia da saúde, medicina psicossomática e psiconeuroimunologia.

No caso da psico-oncologia (ramo da medicina comportamental e psicologia da saúde), o objectivo principal é o de estudar cientificamente os indivíduos com cancro e preocupar-se com as dimensões psicossociais do doente oncológico (Holland, 1989). Segundo Buela-Casal e Moreno (1999), o foco de interesse actual da psico-oncologia centra-se na investigação acerca dos modos de combate à doença oncológica, os factores influentes e as estratégias psicoterapêuticas para melhorar a qualidade de vida dos doentes. Assim, é relevante para a psicologia que a incidência do cancro, bem como a mortalidade prematura a ele associada, possam ser prevenidas por mudanças de comportamento (Andersen, 1989).

O objectivo fundamental da promoção da saúde na doença oncológica é que as diferentes intervenções psicológicas permitam ao doente manter uma boa qualidade de vida e um bem-estar psicológico. Genericamente, as intervenções que se realizam ao longo do percurso da doença são múltiplas e encaminham-se para ajudar o doente a enfrentar a angústia do diagnóstico, os efeitos do tratamento, reduzir a ansiedade, a incerteza, o isolamento, a indefesa, e fomentar uma atitude mais participativa na recuperação posterior e clarificar percepções e infirmações erróneas (Monedo \& Alonso, 2003; Barez, Blaso, \& Castro, 2003). 


\section{A necessidade de intervenções psicossociais na doença oncológica}

Para Justo (2002), o aumento da sobrevida dos doentes oncológicos colocou os técnicos de saúde perante um enorme desafio, a saber: se há cada vez mais sobreviventes das doenças oncológicas, também há mais necessidades de intervenção e mais necessidades de explicação. Segundo o autor, actualmente, assumem especial importância as intervenções que se referem à manutenção ou recuperação da qualidade de vida em múltiplas frentes. Quanto às necessidades de compreensão, temos o facto de a gestão da qualidade de vida só ser eficaz se conseguirmos compreender o papel que o indivíduo desempenhou no aparecimento e desenvolvimento da sua própria doença. Para isso, é proposto o debate sobre a possibilidade de o funcionamento psicológico contribuir para a etiologia das doenças oncológicas (não como alternativa às etiologias biológicas, mas sim como complemento), assim como é importante reflectir sobre o papel dos aspectos psicológicos no surgimento do cancro e, neste sentido, o papel da intervenção psicológica no aumento da eficácia do combate a esta doença, não como alternativa às intervenções médicas, mas como aliada.

Neste âmbito, nas últimas décadas surgiram intervenções psicossociais que foram elaboradas tendo em vista uma melhoria da qualidade de vida dos doentes oncológicos, através de programas terapêuticos diferenciados e focados nos princípios da psicologia da saúde, isto é, encarando o indivíduo numa perspectiva biopsicossocial. Por exemplo, Forester, Kornfeld, Fleiss e Thompson (1993) procuraram estudar os efeitos da terapia de grupo ao nível do stress físico e emocional em doentes com cancro submetidos a radioterapia. O grupo experimental participava na terapia de grupo e era avaliado, antes, durante e no fim do tratamento com radioterapia. Os resultados demonstraram que após algumas semanas depois do término da radioterapia, os sintomas emocionais e físicos tinham sofrido um decréscimo significativamente maior no grupo experimental do que no grupo de controlo. Genericamente, os autores concluíram que a terapia de grupo pode aumentar a qualidade de vida dos doentes durante a radioterapia, e que os doentes oncológicos necessitam partilhar experiências emocionais e sentimentos com outros doentes.

Por sua vez, Evans e Connis (1995) realizaram uma investigação com o intuito de avaliar a eficácia de duas terapias de grupo (terapia cognitivo-comportamental e terapia focada no suporte social) em doentes oncológicos deprimidos a receberem tratamento radioactivo. Os autores constataram que embora ambos os grupos terapêuticos tivessem obtido melhorias em relação ao grupo de controlo (sem terapia de grupo), apenas o grupo que teve sessões de suporte social melhorou significativamente. O follow-up da investigação revelou igualmente que era este último grupo que apresentava efeitos mais duradouros, uma vez que os efeitos positivos se mantinham até seis meses depois, produzindo mudanças favoráveis em mais dimensões relativas ao ajustamento. 
Já Spiegel (1995), ao analisar os dados anteriores, especulou que uma das possibilidades para o grupo de suporte social ter obtido melhores resultados poderia ter a ver com a expressão emocional, tida assim como um efeito terapêutico poderoso, ou seja, a expressão de emoções negativas pode ajudar também a lidar com emoções intensas e desagradáveis. Segundo o mesmo autor, investigações realizadas num laboratório de tratamento psicossocial demonstraram que a supressão emocional é contra produtiva, conduzindo a um maior número de perturbações do humor.

Para Nezu, Nezu, Friedman, Faddis e Houts (1998), um dos problemas mais relevantes que se coloca no combate à doença oncológica está relacionado com os efeitos colaterais dos tratamentos médicos, muitas vezes percepcionados pelo doente como piores do que a própria doença. Esta situação pode levar o doente a confrontar-se com conflitos e dúvidas acerca da utilidade, necessidade de dar continuidade ao sofrimento, limitações e interrogações que interferem na adesão e muitas vezes contribuem para a desistência ao tratamento (Justo, 2002).

Apesar dos conhecimentos alcançados acerca dos sintomas negativos e das medidas farmacológicas desenvolvidas no sentido de combater e minimizar os efeitos adversos dos tratamentos médicos nas doenças oncológicas, os resultados esperados ou ideais nem sempre são conseguidos. No que se refere às náuseas e vómitos, dois dos efeitos adversos mais frequentes e incomodativos para o doente, apesar dos novos fármacos anti-eméticos permitirem reduzir a toxicidade dos tratamentos (Andrews \& Davis, 1993; Bonneterre et al., 1990, cit. por Pereira \& Lopes, 2002), não existe ainda um método eficaz de controlo. São vários os efeitos iatrogénicos nos doentes oncológicos, resultantes quer da doença em si quer dos tratamentos inerentes. No entanto, nem sempre estes efeitos são devidamente diagnosticados. Ao nível da morbilidade psicológica, muitas vezes os problemas não são detectados pelos profissionais de saúde e, segundo os dados de Twycross (1999), cerca de 10\% dos pacientes oncológicos têm uma perturbação mental identificável.

No estudo levado a cabo por Evans e Connis (1995), 80\% dos sujeitos avaliados encontravam-se no ponto de corte para a depressão clínica indicando que a depressão neste doentes poderá constituir a regra e não a excepção. Ou seja, segundo os autores, o que acontece muitas vezes é que os sintomas depressivos são erroneamente atribuídos ao cancro, não se diagnosticando o estado depressivo. Justifica-se assim a tristeza do doente com o facto do prognóstico ser mau; a ansiedade, pelo medo que este tem de morrer; os problemas de sono, derivados do desconforto físico, e a falta de apetite, consequência do tratamento de quimioterapia (Spiegel, 1995). 
Nem todos os doentes reagem bem ao diagnóstico de cancro, sendo as respostas emocionais mais comuns a ansiedade e a depressão, incluindo a expressão de sentimentos de medo, raiva, revolta, desesperança, culpa e pensamentos suicidas (Haynal, Pasin, \& Archinard, 1998). Estas respostas emocionais muitas das vezes são acompanhadas de sintomas físicos de fadiga, perturbações do sono, sexuais e alimentares. Aliás, estudos específicos sobre os níveis de morbilidade psicológica entre os doentes com cancro revelam que a prevalência é muito elevada, desde perturbações da adaptação a outras mais graves (Derogatis, Morrow, Fetting, Penman, Piasetsky, Schmale, Henrichs, \& Carnicke, 1983; Dean \& Hapwood, 1989).

Mclntyre, Fernandes e Pereira (2002) fazem uma importante revisão das intervenções psicossociais de grupo para doentes oncológicos, respectivas avaliações da eficácia, assim como um resumo das principais conclusões importantes para a psicologia da saúde. Genericamente, englobam: (1) intervenções de cariz cognitivo-comportamental, direccionadas particularmente às disfunções cognitivas e aos comportamentos subsequentes, e que procuram a promoção de estratégias adaptativas de coping (Kidman \& Edelman, 1997; Telch \& Telch, 1986, cit. por McIntyre, Fernandes, \& Pereira, 2002); (2) intervenções psicológicas de suporte psicossocial, cuja ênfase é no diálogo com pares e a partilha de informação, no contexto de um grupo que providencia aos seus membros encorajamento e suporte mútuo nas dificuldades induzidas pela doença (Spiegel, Bloom \& Yalom, 1981; Spiegel \& Bloom, 1983; Spiegel et al., 1989; Taylor et al., 1985, cit. por McIntyre, Fernandes, \& Pereira, 2002); (3) intervenções psicológicas para a educação do doente, que enfatizam o controlo da informação através do seu acesso facilitado para que o doente possa responder de forma eficiente às alterações e exigências impostas pela doença (Royak-Schaler, Stanton, \& Danoff-Burg, 1997; Johnston et al., 1992; Jacobs et al., 1983; Pruitt et al., 1993, cit. por McIntyre, Fernandes, \& Pereira, 2002); (4) e intervenções psicológicas multimodais que, de uma forma geral, incorporam componentes de todas as intervenções anteriores. Exemplos deste tipo de intervenção são os trabalhos de Simonton e Simonton (1975), Worden e Weisman (1984), Cain e colaboradores (1986), assim como os de Fawzy e colaboradores (1990, 1994), citados por Mclntyre, Fernandes e Pereira (2002).

Trijsburg, Van Knippenberg e Rijpma (1992) analisaram vários estudos indicativos do impacto positivo das intervenções psicológicas com doentes oncológicos. Os autores referem que a terapia individualizada era particularmente útil para aliviar o distress (stress prejudicial), aumentar a auto-estima e estabelecer um sentido de controlo por parte do doente, para além de aliviar problemas físicos e fomentar um aumento das actividades e relações sexuais dos doentes. Para além disso, os autores verificaram que programas terapêuticos estruturados foram altamente eficazes em termos da redução dos níveis de depressão, do distress, da ansiedade e duma melhoria do auto-conceito. Finalmente, verificaram também que as 
intervenções comportamentais, juntamente com a hipnose, revelaram utilidade na redução da ansiedade, depressão, raiva, hostilidade, confusão e distress, bem como na dor, náuseas e vómitos, relacionados com o tratamento.

O estudo de Andersen (1992) relativo às principais intervenções psicológicas para 258 a melhoria da qualidade de vida em doentes com cancro revela que a terapia possibilita um melhor ajustamento ao cancro, nomeadamente no mantimento das actividades interpessoais, ocupacionais, sociais e sexuais, assim como um alivio dos estados emocionais negativos. De facto, e conforme corroboram Classen e colaboradores (1993, cit. por Trijsburg, Van Knippenberg, \& Rijpma, 1992), ajudar os doentes a adquirirem e a alcançarem comportamentos e atitudes que thes permitam lidar de forma mais eficaz com o cancro pode seguramente melhorar a qualidade das suas vidas, dos seus familiares e o prognóstico da doença.

Nesta linha de pensamento, Pettingale e a sua equipa estudaram as respostas emocionais ao cancro e respectiva influência no curso da doença. Assim, identificaram quatro respostas características ao diagnóstico: I. Espírito de luta; 2. Evitamento positivo (negação); 3. Fatalismo (aceitação estóica); 4. Desesperança/ sensação de incapacidade. Posteriormente, foi adicionado um quinto tipo caracterizado por preocupação ansiosa com pensamentos em relação à doença bem como sentimentos de depressão (Pettingale et al., 1985, cit. por Trijsburg, Van Knippenberg, \& Rijpma, 1992).

Para Pereira e Lopes (2002), as intervenções terapêuticas com doentes oncológicos podem ser úteis não apenas na redução de sintomas específicos mas também na mudança de atitudes e de estilos de personalidade que poderão influir na progressão e adaptação à doença. Ou seja, para as autoras, a ponderação de que as características pessoais podem estar associadas a um bom/mau prognóstico pode representar uma diferença no curso da doença bem como na capacidade para lidar com o diagnóstico e impacto psicossocial.

\section{O mindfulness: O que é e que aplicações tem?}

O mindfulness (ou consciência plena) é uma antiga prática budista que pode ter uma relevância profunda para a vida quotidiana das pessoas (Kabat-Zinn, 1990; 1994). Esta relevância não tem nada a ver com Budismo per se ou em tornar-se budista, tendo sim a ver com o acordar e viver em harmonia consigo mesmo e com o mundo. Tem a ver com o saber quem somos, questionando a nossa visão do mundo e o lugar que ocupamos nele, e com o semear de alguma apreciação da plenitude de cada momento em que estamos vivos. Acima de tudo, tem a ver com o estarmos em sintonia (Germer, 2005). 
O cultivar do mindfulness através da prática da meditação tem uma longa história nas tradições espirituais orientais, principalmente no Budismo (Linehan, 1993a; Kabat-Zinn, 1982). Estas tradições descrevem a meditação mindfulness como um método disponível para reduzir o sofrimento e encorajar o desenvolvimento de qualidades positivas, tais como a consciencialização, insight, sabedoria, compaixão e equanimidade (Goldstein, 2002; Kabat-Zinn, 2003). nossas vidas com acesso a todas as capacidades da nossa consciência, assim como da nossa inconsciência. Segundo Kabat-Zinn (1994), muitos mestres Sages, Yogis e Zen exploraram estes territórios profundamente há milhares de anos. Durante esse processo, eles aprenderam algo que pode ser totalmente benéfico para as populações ocidentais, como contrabalanço das suas orientações culturais que impulsionam para o controlo e subjugação da natureza, em vez de honrarem o facto de serem uma parte constituinte da mesma. O aglomerado das experiências destes mestres sugere que da investigação intensa da nossa própria natureza como seres e, particularmente, da natureza das nossas mentes através de cuidadosas e sistemáticas auto-observações, poderemos capacitar-nos da possibilidade de vivermos as nossas vidas com mais satisfação, harmonia e sabedoria. Esta formulação oferece, igualmente, uma visão do mundo que complementa o reduccionismo predominante e o materialismo que domina o pensamento ocidental, tanto ao nivel individual como institucional. Assim, este ponto de vista não é nem particularmente "oriental" nem místico (Kabat-Zinn, 1990).

O mindfulness foi aclamado como sendo o coração da meditação budista. Fundamentalmente, é até um conceito bastante simples. O seu poder reside na sua prática e nas suas aplicações. Mindfulness significa prestar atenção de uma forma particular, ou seja, no momento presente e sem julgamentos. Esta forma de dirigir a atenção permite uma maior consciencialização, clareza, e aceitação da realidade no momento actual. Desperta-nos para o facto de as nossas vidas se desdobrarem apenas em momentos. Se não estivermos completamente presentes em muitos desses momentos, poderemos não apenas perder o que é mais valioso nas nossas vidas mas também perder a possibilidade de nos apercebermos da profundidade e riqueza das nossas oportunidades de crescimento e transformação (Kabat-Zinn, 1990).

Segundo Bower, Woolery, Sternlieb e Garet (2005), uma consciencialização diminuta do momento presente cria-nos inevitavelmente alguns problemas, manifestos 
através das nossas acções inconscientes e automáticas e dos nossos comportamentos, muitas vezes pautados por medos profundos e inseguranças. Estes problemas tendem a acumular-se com o passar do tempo, se não Ihes dermos a devida atenção, podendo eventualmente levar a um sentimento de bloqueio ou de desrealização. Com esta acumulação, podemos perder a confiança na nossa capacidade de redireccionar as nossas energias para formas de maior satisfação e felicidade, ou até para um melhor estado de saúde (Kabat-Zinn, 2005).

A forma característica como uma pessoa interpreta e reage aos acontecimentos da vida tem influência nas suas atitudes, sentido de auto-eficácia, comportamentos e concretizações. Isto é especialmente saliente quando se lida com uma doença (Tacón, Caldera, \& Ronaghan, 2005). Nas últimas décadas, profissionais de saúde e investigadores ocidentais defenderam que o desenvolvimento da prática do mindfulness poderá ser benéfico para pessoas que sofrem de uma grande margem de problemas ou desordens, mas que não estão interessadas em adoptar uma terminologia ou tradições budistas (Baer \& Krietemeyer, 2006).

Para Kabat-Zinn, Massion, Hebert e Rosenbaum (1998), o mindfulness providencia uma via simples mas poderosa para nos desbloquearmos, de voltar para a sintonização com a nossa própria sabedoria e vitalidade. É uma forma de assumirmos a direcção e qualidade das nossas próprias vidas, incluindo os nossos relacionamentos familiares, o trabalho, assim como para com o mundo e planeta, mas fundamentalmente, para o relacionamento connosco mesmos. A chave para este caminho que, como foi dito, resulta das raízes do Budismo, Taoismo, e Yoga, é uma apreciação do momento presente e o cultivar de um relacionamento íntimo com ele de uma forma contínua e discernida. É completamente o oposto de conceptualizar a vida como um bem adquirido.

O hábito de ignorarmos os nossos momentos presentes em detrimento de outros conduz a uma constante falta de consciência da rede que é a vida na qual estamos embrenhados. Isto inclui a falta de consciência e compreensão da nossa própria mente e como isso influencia as nossas percepções e acções. Isso limita severamente a nossa perspectiva do que é ser pessoa e como estamos ligados uns aos outros e ao mundo à nossa volta (Kabat-Zinn, 2005). Tradicionalmente, a religião tem tido domínio sobre tais questões fundamentais com uma armação espiritual, mas o mindfulness tem pouco a ver com religião, excepto no significado mais fundamental da palavra, como uma tentativa de apreciar o profundo mistério de estar vivo e tomar conhecimento, e de estar vitalmente ligado a tudo o que existe (Olendzki, 2005).

Quando nos comprometemos a prestar atenção de uma forma aberta, sem estarmos presos às nossas preferências, opiniões e preconceitos, projecções e expectativas, abrimos oportunidades de nos libertarmos da prisão que pode ser 
a inconsciência. O mindfulness não interfere com crenças ou tradições - religiosas ou relacionadas em termos científicos - nem tenta vender nada, muito menos um novo sistema de crenças ou ideologia. É simplesmente uma forma prática de estar mais em contacto com a plenitude do ser, através de um processo sistemático de auto-observação, auto-questionamento e acção preenchida de consciência. De uma forma abstracta, o praticante mor do mindfulness é gentil, contemplativo e dedicado. Assim, outra maneira de definir mindfulness será "heartfulness" (Kabat-Zinn et al., 1998).

\section{Mindfulness como construto}

O mindfulness como construto é o ponto de encontro entre várias intervenções clínicas. Tentativas recentes de definição resultaram num modelo de dois componentes do mindfulness (Bishop, Lau, Shapiro, Carlson, Anderson, Carmody, Segal, Abbey, Speca, Velting, \& Devins, 2004), dos quais o primeiro envolve a auto-regulação da atenção na experiência imediata, resultando na recognição de acontecimentos que surgem no momento presente da consciência. O segundo componente representa uma orientação de abertura e aceitação da experiência momento-a-momento. Isto é similar com a definição do mindfulness como sendo constituído por intenção, atenção, e atitude (Shapiro, Bootzin, Figueiredo, Lopez, \& Schwartz, 2003). A intenção neste modelo descreve a natureza intencional de dirigir as faculdades da atenção para observar a experiência momento-a-momento, paralelamente à primeira componente tal como descrita por Bishop e colaboradores (2004). $\mathrm{O}$ terceiro atributo, a atitude, descreve a qualidade da atenção, a sua suavidade, aceitação sem julgamentos do que quer que surja no campo da consciência, similar à segunda componente conforme descrita pela equipa de Bishop (2004). Apesar disso, em ambas as conceptualizações, o mindfulness envolve não apenas o prestar atenção, mas o fazê-lo de uma forma que seja acompanhada de uma atitude de não-julgamento e aceitação aberta.

O mindfulness permite ao praticante exercitar um método activo de desligar a reactividade evasiva que é comum nas vidas dos seres ocidentais e substitui-la por respostas mais conscientes (Kabat-Zinn, 1994).

O mindfulness é cultivado pela prática de várias formas de meditação, ou treino mental. Pode ser executado em sessões de meditação formal ou durante as actividades do dia-a-dia, tais como lavar pratos, simplesmente estando presente no momento que permite à pessoa experienciá-lo na sua plenitude e totalidade (Kabat-Zinn, 1994). 


\section{Mindfulness e tratamento da doença oncológica}

Conceptulizando a prática da meditação mindfulness tradicional como um conjunto de capacidades que podem ser aprendidas independentemente de qualquer sistema de crenças religiosas, investigadores e clínicos tornaram o treino em mindfulness disponivel para as populações ocidentais, incorporando-o em intervenções que estão a ter um crescendo significativo em contextos médicos e de saúde mental (Baer \& Krietemeyer, 2006). Genericamente, estas intervenções englobam: (1) Redução do stress baseada no mindfulness (MBSR) (Kabat-Zinn, 1982, 1990); (2) Terapia cognitiva baseada no mindfulness (MBCT) (Segal, Williams, \& Teasdale, 2002); (3) Terapia comportamental-dialéctica (DBT) (Linehan, 1993a; 1993b); e (4) Terapia da aceitação-compromisso (ACT) (Hayes, Strosahl, \& Wilson, 1999).

Quase metade de todos os doentes com cancro sofre de distress psicológico moderado e severo, e enfrentam dificuldades consideráveis no que concerne às estratégias de coping relacionadas com a doença (Carlson, Angen, Cullum, Goodey, Koopmans, Lamont, MacRae, Martin, Pelletier, Robinson, Simpson, Speca, \& Bultz 2004; Spiegel, 1996; Zabora, BrintzenhofeSzoc, Curbow, Hooker, \& Piantadosi, 2001). Existem muitas fontes potenciais de distress para pessoas diagnosticadas com um cancro, incluindo a antecipação do sofrimento, as exigências dos tratamentos, dificuldades em aceitar as mudanças na vida, e adaptar-se à incerteza e incontrolabilidade inerentes da doença (Carlson, Ursuliak, Goodey, Angen, \& Speca, 2001).

Os tratamentos habituais do cancro, que incluem a cirurgia, a radioterapia, a quimioterapia, e hormonoterapia, objectivam a remoção, retardamento ou aniquilação das células tumorais. Os oncologistas e outros profissionais de saúde podem também recomendar outros tipos de tratamento perspectivados para produzir melhorias na saúde geral e no bem-estar. Estas intervenções são frequentemente referidas como complementares, dado serem utilizadas paralelamente às terapias habituais (Hilsden \& Verhoef, 1999; Verhoef, Hilsden, \& O'Beirne, 1999). Para os doentes que usam terapias complementares, tomar decisões no sentido de usarem estas terapias providencia uma oportunidade significativa de obter algum controlo sobre a sua doença, tratamento e bem-estar durante os tratamentos e recuperação do cancro (Truant \& Bottorff, 1999; Verhoef, Hilsden, \& O’Beirne, 1999).

A meditação mindfulness é um exemplo de uma terapia complementar que tem sido usada por doentes oncológicos assim como praticantes na área da medicina comportamental (Baer, 2003; Bishop, 2002). Têm surgido cada vez mais programas de tratamento clínico e de promoção do bem-estar baseados na meditação mindfulness e no yoga, modelados a partir do programa de redução de stress baseado no mindfulness (Mindfulness-Based Stress Reduction - MBSR) de John Kabat-Zinn e seus colegas na Clínica de Redução do Stress do Centro Médico da Universidade de Massachusetts, nos Estados Unidos. 
Doentes com condições médicas crónicas que participaram em programas de MBSR demonstraram obter mudanças positivas nos seus estados de saúde físico e mental (Bishop, 2002; Grossman, Niemann, Schmidt, \& Walach, 2004). Os efeitos de promoção da saúde do MBSR parecem complementar os tratamentos biomédicos convencionais, numa abordagem compreensiva, centrada no paciente, acerca do alivio e cura do sofrimento humano (Reibel, Greeson, Brainard, \& Rosenzweig, 2001).

\section{A aplicação de programas de MBSR em populações oncológicas: resultados actuais}

O aumento da qualidade de vida, relacionada com a saúde, e com melhorias ao nível da vitalidade, com menos dor corporal, menos limitações provocadas pela saúde física, melhor funcionamento social, e diminuições dos níveis de ansiedade e depressão foram demonstrados num grupo de doentes com diagnósticos médicos diversificados, sujeitos a um programa baseado nos propostos terapêuticos do mindfulness (Reibel et al., 2001). Tendo em conta estes resultados promissores, é natural que os investigadores se tenham debruçado sobre as características da doença oncológica e o seu estudo em termos de evolução tendo em conta a prática de técnicas de mindfulness, principalmente porque é sobejamente conhecido o stress emocional após a tomada de conhecimento do diagnóstico de cancro (Strain, 1998; Zabora, Blanchard, Smith, Roberts, Glajchen, Sharp, Brintzenhofe-Szoc, Locher, Carr, Best-Castner, Smith, Dozier-Hall, Polinsky, \& Hedlund, 1997).

Existe actualmente na população de doentes oncológicos um interesse crescente na medicina corpo-mente e nas terapias complementares, sustentado por um desejo de ser proactivo e de tomar iniciativas no tratamento pessoal (Weiger, Smith, Boon, Richardson, Kaptchuk, \& Eisenberg, 2002). Por exemplo, quando perguntadas acerca da causa do seu cancro da mama, uma amostra de mulheres canadianas citaram "stress" a cima de qualquer outro factor possivel, como genéticos, dietéticos, e factores ambientais (Stewart, Cheung, Duff, Wong, McQuestion, Cheng, Purdy, \& Bunston, 2001).

O MBSR providencia não apenas um tratamento eficaz para o stress, enquadrando-se também na própria estrutura pessoal do doente no que diz respeito a comportamentos de saúde positivos (Kabat-Zinn et al., 1998; Rosenbaum, 2005).

Uma revisão da literatura efectuada por Ott, Norris e Bauer-Wu (2006) revela cerca de sete estudos que investigaram a eficácia das intervenções com programas de relaxamento e da redução do stress baseados no mindfulness em doentes com cancro. Três destes estudos focaram-se em mulheres com cancro da mama (Shapiro et al., 2003; Tacón, Caldera \& Ronaghan, 2004, 2005), o último dos quais investigando 
os factores psicossociais. Speca, Carlson, Goodey e Angen (2000) investigaram os resultados do mesmo programa numa amostra heterogénea de doentes oncológicos, procurando avaliar os efeitos ao nível dos estados de humor e sintomatologia de stress. Os participantes neste estudo demonstraram uma redução significativa nas perturbações do humor (65\%) e em todos os níveis gerais de stress (31\%), quando comparados com o grupo de controlo. Estes benefícios persistiram no follow-up após 6 meses, conforme demonstrado por Carlson e colaboradores (2001). Os resultados obtidos estão em concordância com outros estudos publicados que confirmam a efectividade do treino mindfulness na tranquilidade e na redução de sintomas físicos e psicológicos associados à doença numa variedade de populações clínicas, tais como: dor crónica (Kabat-Zinn, 1982; Kabat-Zinn, Lipworth, Burney, \& Sellers, 1986) perturbações da ansiedade (Kabat-Zinn, Massion, Kristeller, Peterson, Fletcher, Pbert, Lenderking, \& Santorelli, 1992; Miller, Fletcher, \& Kabat-Zinn, 1995), fibromialgia (Kaplan, Goldenberg, \& Galvin-Nadeau, 1993; Singh, Berman, Hadhazy, \& Creamer 1998), epilepsia (Deepak, Manchanda, \& Maheshwari, 1994), psoríase (Kabat-Zinn, Wheeler, Light, Skillings, Scharf, Cropley, Hosmer, \& Bernhardt, 1998), e hipertensão (Schneider, Staggers, Alexander, Sheppard, Rainforth, Kondwani, Smith, \& King, 1995). O sexto estudo relativo ainda à doença oncológica faz uma revisão do impacto do programa numa amostra heterogénea de doentes oncológicos, relativamente aos sintomas de fadiga, stress, estados de humor e qualidade do sono (Carlson \& Garland, 2005), e o sétimo relaciona o mesmo programa com a qualidade de vida de doentes com cancro da mama e da próstata, assim como com sintomas de stress, estados de humor e parâmetros imunitários (Carlson, Speca, Patel, \& Goodey, 2003). O diagnóstico, tratamento, e a sobrevivência ao cancro estão associados a uma variedade de consequências psicológicas e físicas. Conforme sustentam diferentes investigações transversais e longitudinais, as técnicas de redução do stress podem ser incorporadas no cuidado de pacientes com cancro para promover um coping eficaz, para diminuir sintomas, e para melhorar estados de saúde. Deste modo, as intervenções baseadas no mindfulness que contemplam a relação mente-corpo estão a torna-se cada vez mais populares e a ganhar credibilidade para o uso nas populações oncológicas. Para Ott, Norris e Bauer-Wu (2006), embora este campo de investigação esteja ainda a emergir, com um primeiro estudo publicado em 2000, as pesquisas até à data demonstram benefícios notáveis de intervenções baseadas no mindfulness que realçam o bem-estar das pessoas que vivem e lidam com o cancro.

Apesar de tudo, o corpo de investigações empíricas que suportam o uso do MBSR em doentes com cancro está a crescer, embora de uma forma preliminar. De acordo com Bishop (2002), apesar da eficácia do MBSR na autogestão do stress e de sintomas de humor associados com o cancro parecer particularmente promissora, será complicado recomendar esta aplicação como modelo de intervenção, baseando-se 
apenas numa única experiência controlada aleatoriamente: "The Tom Baker study". Embora esta experiência seja importante porque representa o primeiro estudo rigoroso da eficácia desta abordagem, Bishop aconselha que deverá ser aplicado de uma forma mais consistente até poder ser recomendado para uma maior margem de populações médicas. Assim, a replicação dos estudos é fundamental para estabelecer a eficácia deste programa nas populações oncológicas. Para além das replicações dos estudos de eficácia, são necessários estudos que comparem o MBSR com outras intervenções psicossociais, assim como pesquisas desenhadas para explicar as contribuições relativas dos vários componentes desta intervenção multifacetada (Speca, Carlson, MacKenzie, \& Angen, 2006).

\section{Efeitos do mindfulness ao nível do sistema imunitário}

O stress é um dos factores que surge frequentemente associado ao tratamento do cancro, podendo mesmo ser uma consequência dele e até dificultar a sua eficácia (Evans, Hucklebridge, \& Clow, 2000). O modelo biocomportamental defende que reduzindo o stress aumentamos a capacidade do sistema imunitário para dar resposta à doença, daí a necessidade de actuar ao nível dos comportamentos de saúde de modo a aumentar a adesão aos tratamentos e a qualidade de vida dos doentes (Anderson, Kiecolt-Glaser, \& Glaser, 1994; Fawzy \& Fawzy, 1994). Para além disso, e segundo Telch e Telch (1986, cit. por Pereira \& Lopes, 2002), fornecer ao doente competências de resolução de problemas, treino de relaxamento e planeamento de actividades apraziveis parece ter um efeito benéfico ao nível da mortalidade psicológica, adesão terapêutica, comunicação e relacionamento social.

Para além dos efeitos psicológicos demonstrados pela prática da redução do stress baseada no mindfulness, estudos demonstram que a prática da meditação tem sido relacionada com efeitos ao nível do sistema imunológico. Solberg e colaboradores (1995), descobriram que a meditação pode modificar a influência supressora do stress do exercício físico vigoroso no sistema imunitário de atletas masculinos. Um estudo casual com treino em relaxamento, meditação, e hipnose em homens com VIH-positivo descobriu melhorias na contagem das células T no grupo de tratamento, e que se mantiveram após um mês de follow-up (Taylor, 1995). Também outras técnicas, como o QiGong, um sistema energizante chinês que combina técnicas meditativas com outras práticas, estava associado com a elevação das células T CD4 e um aumento proporcional das células CD4/CD8 num grupo de praticantes saudáveis comparativamente com um grupo de indivíduos saudáveis do grupo de controlo (Ryu et al., 1995).

Muitos estudos demonstram que os doentes oncológicos têm uma função imunitária comprometida (Andersen, Kiecolt-Glaser, \& Glaser, 1994), e factores imunitários 
têm sido usados para predizer a progressão da doença (Kiecolt-Glaser, McGuire, Robles, \& Glaser 2002a, 2002b). Por exemplo, Levy, Herberman, Lippman, D’Angelo e Lee (1991) descobriram que menos indicadores de stress, num inventário relativo à avaliação do perfil do humor, previram intervalos maiores sem doença em doentes com cancro da mama, e menos células NK previram um reaparecimento. De qualquer forma, nenhuma intervenção de carácter psicossocial foi efectuada neste género de estudos.

Adicionalmente a estes estudos mais observacionais, estudos com recurso a intervenções investigaram os efeitos das terapias em medidas do sistema imunitário, inicialmente em doentes com cancro da mama. Os estudos de Fawzy e colaboradores (1990, cit. por Mclntyre, Fernandes, \& Pereira, 2002) são disso um exemplo, tendo os autores estruturado uma intervenção psicossocial de seis semanas para pacientes com melanoma maligno e tendo encontrado aumentos significativos na percentagem dos grandes linfócitos e das células NK, indicações de aumentos na actividade das NK citotóxicas, e uma pequena redução na percentagem das células T CD4 (helper). Estas mudanças não foram observadas imediatamente após a intervenção, mas evidenciaram-se numa avaliação de follow-up após seis meses. Segundo Justo (2002), o aspecto mais importante dos dados a presentados pela equipa de Fawzy é esta indicação de que os frutos da intervenção psicológica continuam activos e produtivos depois de terminada a intervenção. Contudo, e como também alerta o mesmo autor, nem todos os estudos sobre efeitos imunitários das intervenções encontraram melhorias nas funções imunitárias, pelo que os resultados deverão ser interpretados com algumas reservas.

Segundo Carlson e colaboradores (2003), para além do deles, não há qualquer estudo publicado até à data que investigue os efeitos do programa de redução do stress baseado no mindfulness na função imunitária dos doentes com cancro. Conforme os autores, os moldes em que as mudanças no sistema imunitário traduzem uma progressão ou uma regressão da doença estão actualmente a ser debatidos na literatura, conforme corroboram os estudos de Spiegel, Sephton, Terr e Sittes (1998), assim como os de Garssen e Goodkin (1999). Aliás, estes últimos questionam a importância da imuno-modulação da progressão do cancro, sugerindo que talvez outras medidas, tais como a activação endócrina, poderão ser mais importantes. Contudo, os resultados promissores da equipa de Carlson (2003) fazem referência às seguintes alterações nos elementos da amostra onde se aplicou o programa: as mudanças ao nível do sistema imunitário dos doentes foram consistentes e comportamentalmente associadas a uma alteração dos padrões depressivos com uma funcionalidade imunitária mais saudável. Apesar das alterações terem sido moderadas, os autores atribuem esse facto aos altos níveis de funcionamento psicológico e imunitário dos doentes no início do estudo. 
Sumariamente, para Kiecolt-Glaser, Robles, Heffner, Loving e Glaser (2002), existem evidências substanciais em populações saudáveis e populações com doenças oncológicas que relacionam stress psicológico com desregulação imunológica. Segundo os autores, o stress pode potenciar carcinomas através de alterações na reparação do ADN e/ou apoptose (morte celular programada). Para além disso, argumentam que a possibilidade de as intervenções psicológicas poderem melhorar a função imunitária e a sobrevida em doentes oncológicos é ainda uma pergunta em aberto, assim como a sugestão de o suporte social poder ser um mediador psicológico chave. No entanto, os autores defendem que os estudos permitiram concluir que factores psicológicos e comportamentais podem influenciar o despoletar e a progressão do cancro.

\section{Questões práticas do MBSR}

Torna-se evidente de que o apoio psicológico ao doente oncológico no contexto de grupo tem benefícios alargados em termos de qualidade de vida do doente, repercutindo-se quer no seu bem-estar físico, quer no psicossocial.

O formato da intervenção MBSR, descrita por Kabat-Zinn (1982, 1996, 2003), é o de uma actividade psico-educativa que se realiza em grupo, constando de nove sessões num total de 28 horas presenciais, repartidas por oito semanas. As sessões são semanais e duram cerca de 2,5 horas, excepto na sexta semana, quando para além da sessão semanal há também uma sessão especial de oito horas, ou dia intensivo. As sessões semanais incluem a apresentação de um tema, exercícios práticos, e tempo de debate em grupo acerca das melhorias e das dificuldades que surgiram na aplicação das técnicas e das estratégias de coping na vida diária.

Especificamente, ao longo do programa, é efectuado treino em três práticas formais do mindfulness: o body scan, a meditação sentada e o yoga: a) O body scan, ou yoga deitado, inclui uma mudança gradual do foco de atenção por todo o corpo, da cabeça aos pés, de uma forma não-crítica, em todas as sensações e sentimentos das regiões corporais, com sugestões periódicas acerca da consciencialização na atenção e no relaxamento; b) A meditação sentada envolve a atenção mindfulness da respiração e outras percepções, promovendo uma consciência observacional e sem julgamentos das cognições e das correntes de pensamento e distracções que passam constantemente pela mente; c) O yoga envolve um conjunto de estiramentos simples e posturas estudadas para relaxar e alongar o sistema músculo-esquelético, e desenvolver movimentos em mindfulness acerca da relação corpo-meditação (Tacón, Caldera, \& Ronaghan, 2005). 
O programa das sessões realizadas é o seguinte (cf. Martín \& García de la Banda, 2007):

(1) A consciência plena. Nesta sessão é apresentada a ideia de mindfulness nos pensamentos, emoções e sensações, incidindo na importância de parar e ver, antes de agir, e incentiva-se os participantes a experimentarem pessoalmente este tipo de consciencialização. Assim, é realizado um exercício prático de exploração das sensações do corpo, fornecendo-se um CD para a continuação da prática pessoal.

(2) As percepções e a realidade. São explicadas as características da percepção e o efeito do condicionamento prévio incidindo-se na ideia de responder em vez de reagir perante os desafios da vida e os momentos de stress. Nesta sessão pratica-se novamente o exercício de exploração do corpo e introduz-se a meditação vipassana (que significa ver as coisas como realmente são, sendo uma das mais antigas técnicas de meditação da Índia), de atenção centrada na respiração.

(3) As emoções. É feita uma introdução às emoções básicas (medo, raiva, tristeza e alegria) e são analisadas as suas características a partir da consciência plena. É explicado o efeito das emoções na vida diária e nas relações pessoais. Nesta sessão introduz-se o yoga deitado (body scan) e é fornecido um CD com exercícios de yoga para praticar entre as sessões.

(4) O stress, reacção e resposta. São apresentadas as bases de compreensão do stress e indicadas as características da resposta ao stress com recurso ao modelo biopsicossocial. Pratica-se novamente yoga deitado e atenção na respiração.

(5) Estratégias para enfrentar o stress. São explicados os tipos de estratégias de coping e as suas características, assim como os perigos associados ao stress. É praticado yoga em pé (incluído numa gravação) e atenção à respiração.

(6) A comunicação e a alimentação. Nesta sessão são analisados os efeitos da alimentação e da comunicação no stress e na saúde, observados através da consciência plena. Pratica-se yoga de pé e amplia-se o tempo de meditação com um novo CD que inclui uma prática guiada, e pratica-se meditação caminhando.

(7) Dia intensivo. Jornada destinada à prática, mas que se realiza em silêncio excepto nas indicações dadas pelo instrutor. São realizadas todas as técnicas introduzidas de yoga e meditação e no final da jornada volta-se a usar a palavra para debater em grupo as experiências da jornada. Por último, e em silêncio novamente, pratica-se a consciência plena.

(8) A gestão do tempo. Nesta sessão são apresentadas as bases da gestão de tempo na óptica da consciência plena orientadas para pessoas com stress. Seguidamente, pratica-se de novo a meditação guiada. 
(9) Despedida. Os participantes são incentivados a integrar estas aprendizagens nas suas vidas, e realiza-se uma roda de comentários entre os assistentes sobre a sua experiência no programa. Para além disso, a sessão é aproveitada para praticar a exploração do corpo e a meditação guiada.

O programa MBSR delineado para populações oncológicas procura corresponder à maximização dos benefícios deste tipo de intervenção. Para a população oncológica, pretende-se então que o programa MBSR se revista de um carácter multimodal contemplando, na nossa perspectiva, os seguintes componentes:

(1) Educação para a saúde. Os participantes aprendem as principais relações entre o stress, o cancro, e os factores do estilo de vida. Para tal é fornecido material teórico e prático acerca do mindfulness, relaxamento, meditação, yoga, e da relação mente-corpo;

(2) Gestão do stress. Através da prática experimental de meditação e yoga durante os encontros do grupo e na prática em casa, os participantes são ensinados a prestar atenção às sensações físicas e aos efeitos do stress e ansiedade. Ou seja, são conduzidos a detectar os sinais fisiológicos do stress no corpo para depois os dissiparem com recurso a estratégias cognitivas e sensoriais.

(3) Promoção do coping. Utilização dos processos grupais focados na resolução de problemas, nos métodos de comportamento e pensamento activo, e na consideração dos impedimentos para a prática efectiva, exercícios diários das aplicações do mindfulness, e interacção de suporte entre os membros do grupo;

(4) Gestão emocional. Consciencialização progressiva de que existem coisas específicas que podem ser feitas quando os sintomas ameaçam surgir devido à doença. Cada participante aprende a identificar os seus próprios sinais, embora seja fomentado que podem ajudar-se uns aos outros fazendo planos sobre a melhor forma de responder a estes sinais. É transmitido aos participantes que a prática regular do mindfulness ajuda a manter o equilíbrio na vida, isto é, que as boas intenções podem ser fortalecidas através da união dessas intenções com uma razão positiva para tomar conta de si mesmo. O objectivo último da gestão emocional é que os participantes consigam pensar e praticar consigo mesmos exercícios de auto-compaixão.

Os facilitadores, ou instrutores, da intervenção MBSR deverão possuir qualificações relativamente específicas de treino em MBSR num contexto de oncologia, devendo estas incluir uma combinação a propriada de treino em MBSR e experiência relevante supervisionada como facilitador de grupos semelhantes, bem como alguma familiaridade com os desafios enfrentados pelos doentes com cancro. Os facilitadores deverão assumir um compromisso contínuo de manter uma prática pessoal de mindfulness de forma a assegurar a credibilidade experiencial numa 
base segura de maneira a poder responder às questões e necessidades dos participantes (Speca, Carlson, MacKenzie, \& Angen, 2006).

Para além disso, os restantes facilitadores serão cada elemento do grupo, dado que o programa enfatiza o intercâmbio experiencial. No que diz respeito ao contexto de aplicação, o programa foi delineado para ser implementado em instituições de saúde.

\section{Conclusões}

A prática clínica em todas as disciplinas relacionadas com a saúde está cada vez mais a ser moldada pelo imperativo ético de que as evidências da eficácia das intervenções oferecidas aos doentes serão desenvolvidas (Grol \& Grimshaw, 2003). Em 1998, Kabat-Zinn e os seus colaboradores apresentam pela primeira vez a possibilidade da meditação poder ser utilizada em doentes oncológicos, e testada cientificamente (Kabat-Zinn et al., 1998). Desde então, pouco mais de meia-dúzia de investigações foram realizadas nesse intuito. Contudo, conforme alertam Speca, Carlson, MacKenzie e Angen (2006), na área da oncologia deverão ser consideradas de uma forma sensata as possibilidades de integração do programa de investigação e avaliação do programa de MBSR.

Segundo os investigadores que efectivamente praticaram o programa MBSR com populações oncológicas, devem ser ponderadas as oportunidades para informar os profissionais de saúde e os administradores acerca da natureza desta intervenção, o seu papel auxiliar ou complementar relativamente ao tratamento do cancro, e os benefícios potenciais para os doentes (Speca, Carlson, MacKenzie, \& Angen, 2006). Isto é, nos contextos de implementação do MBSR deverão ser considerados dois aspectos: (1) o acesso aos doentes, e (2) o assegurar da receptividade daqueles que possam estar numa posição de referenciar doentes ou providenciar outras formas de programas de suporte.

Profissionais de saúde estabelecidos nas disciplinas tradicionais, tais como a psicologia, a medicina, e a enfermagem que já estejam a trabalhar com doentes oncológicos e que tenham obtido treino específico no MBSR, poderão estar numa situação ideal para introduzir as práticas do mindfulness nos contextos da oncologia. Para isso, será fundamental a conceptualização dos cuidados e tratamentos aos doentes oncológicos em Portugal num modelo biopsicossocial dos cuidados, tendo em consideração as técnicas do mindfulness como uma componente de uma abordagem integrada e multidisciplinar do tratamento do cancro. 


\section{Referências Bibliográficas}

Andersen, B. L. (1989). Health psychology's contribution to addressing the cancer problem: Update on accomplishments. Health Psychology, 8, 683-703.

Andersen, B. L. (1992). Psychological interventions for cancer patients to enhance the quality of life. Journal of Consulting and Clinical Psychology, 60, 552-568.

Andersen, B., Kiecolt-Glaser, J. \& Glaser, R. (1994). A biobehavioral model of cancer stress and disease course. American Psychologist, 49, 389-404.

Baer, R. \& Krietemeyer, J. (2006). Overview of mindfulness and acceptance-based treatment approaches. In R. A. Baer (Ed.), Mindfulness-based treatment approaches: Clinician's guide to evidence base and applications (pp. 3-27). London: Academic Press.

Baer, R. A. (2003). Mindfulness training as clinical intervention: A conceptual and empirical review. Clinical Psychology: Science and Practice, 10, 125-143.

Barez, M., Blaso, T. \& Castro, F. (2003). La inducción de sensación de control como elemento fundamental de la eficacia de las terapias psicológicas en pacientes de cáncer. Anales de Psicologia, 9, 235-236.

Barreto, P., Ferrero, J. \& Toledo, M. (1993). La intervención psicológica en pacientes de cáncer. Clínica y Salud, 43, 253-262.

Bishop, S. R. (2002). What do we really know about mindfulness-based stress reduction? Psychosomatic Medicine, 64, 71-83.

Bishop, S., Lau, M., Shapiro, S., Carlson, L., Anderson, N., Carmody, J., Segal, Z., Abbey, S., Speca, M., Velting, D. \& Devins, G. (2004). Mindfulness: A proposed operational definition. Clinical Psychology: Science and Practice, 11, 230-241.

Bower, J., Woolery, A., Sternlieb, B. \& Garet, D. (2005). Yoga for cancer patients and survivors. Cancer Control, 12, 165-171.

Buela-Casal, G. \& Moreno, S. (1999). Intervención psicológica en cancer. In M. A. Simón (Ed.), Manual de Psicologia de la Salud. Fundamentos, Metodologia y Aplicaciones (pp. 505-535). Madrid: Editorial Biblioteca Nueva.

Carlson, L. \& Garland, S. (2005). Impact of mindfulness-based stress reduction (MBSR) on sleep, mood, stress and fatigue symptoms in cancer outpatients. International Journal of Behavioral Medicine, 12, 278-285.

Carlson, L., Speca, M., Patel, K. \& Goodey, E. (2003). Mindfulness-based stress reduction in relation to quality of life, mood, symptoms of stress, and immune parameters in breast and prostate cancer outpatients. Psychosomatic Medicine, 65, 571-581.

Carlson, L., Ursuliak, Z., Goodey, E., Angen, M. \& Speca, M. (2001). The effects of a mindfulness meditation-based stress reduction program on mood and symptoms of stress in cancer outpatients: 6-month follow-up. Supportive Care in Cancer, 9, 112-123.

Carlson, L.E., Angen, M., Cullum, J., Goodey, E., Koopmans, J., Lamont, L., MacRae, J. H., Martin, M., Pelletier, G., Robinson, J., Simpson, J. S., Speca, M., \& Bultz, B. D. (2004). High levels of untreated distress and fatigue in cancer patients. British Journal of Cancer, 9o, 2297-2304

Dean, C. \& Hapwood, P. (1989). Liaison psychiatry in a breast cancer unit: Comment. British Journal of Psychiatry, 155, 98-100.

Deepak, K., Manchanda, S. \& Maheshwari, M. (1994). Meditation improves clinicoelectroencephalographic measures in drug-resistant epileptics. Biofeedback Self Regulation, 19, 25-40. 
Derogatis, L. R., Morrow, G. R., Fetting, J., Penman, D., Piasetsky, S., Schmale, A. G., Henrichs, M. \& Carnicke, C. L., (1983). The prevalence of psychiatric disorders among cancer patients. Journal of the American Medical Association, 249, 751-757.

Evans, P., Hucklebridge, F. \& Clow, A. (2000). Mind, immunity and health: The science of psychoneuroimmunology. London: Free Association Books.

Evans, R.L. \& Connis, R. T. (1995). Comparison of brief group therapies for depressed cancer patients receiving radiation treatment. Public Health Report, 110, 306-311.

Fawzy, I. F. \& Fawzy, N. W. (1994). Psychoeducational interventions and health outcomes. In R. Glaser \& J. Kiecolt-Glaser (Eds.), Handbook of human stress and immunity (pp. 365-402). London: Academic Press.

Forester, B., Kornfeld, D., Fleiss, J. \& Thompson, S. (1993). Group psychotherapy during radiotherapy: Effects on emotional and physical distress. American Journal of Psychiatry, 150, 1700-1706.

Fulton, P. \& Siegel, D. (2005). Buddhist and Western psychology: Seeking common ground. In C. K. Germer, R. D. Siegel \& P. R. Fulton (Eds.), Mindfulness and psychotherapy (pp. 28-54). New York: Guilford.

Garssen, B. \& Goodkin, K. (1999). On the role of immunological factors as mediators between psychosocial factors and cancer progression. Psychiatry Research, 85, 51-61.

Germer, C. K. (2005). Mindfulness: What is it? What does it matter? In C. K. Germer, R. D. Siegel \& P. R. Fulton (Eds.), Mindfulness and psychotherapy (pp. 3-27). New York: Guilford.

Goldstein, J. (2002). One dharma: The emerging Western Buddhism. San Francisco: HarperCollins.

Gorin, S. S. (1998). Models of health promotion. In S. S. Gorin \& J. Arnold (Coords.), Health promotion handbook (pp. 14-38). London: Mosby.

Grol, R., \& Grimshaw, J. (2003). From best evidence to best practice: Effective implementation of change in patients' care. Lancet, 362, 1225-1230.

Grossman, P., Niemann, L., Schmidt, S., \& Walach, H. (2004). Mindfulness-based stress reduction and health benefits. A meta-analysis. Journal of Psychosomatic Research, 57, 35-43.

Hayes, S. C., Strosahl, K., \& Wilson, K. G. (1999). Acceptance and commitment therapy. New York: Guilford Press.

Haynal, A., Pasini, W. \& Archinard, M. (1998). Medicina psicossomática: Perspectivas psicossociais. Lisboa: Climepsi.

Hilsden, R.J., \& Verhoef, M.J. (1999). Complementary therapies: Evaluating their effectiveness in cancer. Patient Education and Counseling, 38, 101-108.

Holland, J. C. (1989). Behavioral and psychosocial risk factors in cancer: Human studies. In J. C. Holland \& J. H. Rowland (Eds.), Handbook of Psycho-Oncology. New York: Oxford University Press.

Justo, J. M. (2002). Uma perspectiva psicológica sobre as doenças oncológicas: Etiologia, intervenção e articulações. In M. R. Dias \& Durá, E. (Coords), Territórios da psicologia oncológica (pp. 51-74). Lisboa: Climepsi.

Kabat-Zinn, J. (1982). An outpatient program in behavioral medicine for chronic pain patients based on the practice of mindfulness meditation: Theoretical considerations and preliminary results. General Hospital Psychiatry, 4, 33-42.

Kabat-Zinn, J. (1990). Full catastrophe living: using the wisdom of your body and mind to face stress, pain, and illness. New York: Dell Publishing.

Kabat-Zinn, J. (1994). Wherever you go, there you are: Mindfulness meditation in everyday life. New York: Hyperion. 
Kabat-Zinn, J. (1996). Mindfulness meditation, what is what isn't. In Y. Haruki, Y. Ishii \& M. Suzuki (Eds.), Comparative and psychological study on meditation (pp. 161-170). Netherlands: Eburon.

Kabat-Zinn, J. (2003). Mindfulness-based interventions in context: Past, present, and future. Clinical Psychology: Science and Practice, 10, 144-156.

Kabat-Zinn, J. (2005). Coming to our senses: Healing ourselves and the world through mindfulness. New York: Hyperion.

Kabat-Zinn, J., Lipworth, L., Burney, R. \& Sellers, W. (1986). Four-year follow-up of a meditation based program for the self-regulation of chronic pain: Treatment outcomes and compliance. Clinical Journal of Pain, 2, 159-173.

Kabat-Zinn, J., Massion, A., Kristeller, J., Peterson, L., Fletcher, D., Pbert, L., Lenderking, W. \& Santorelli, S. (1992). Effectiveness of a meditation-based stress reduction program in the treatment of anxiety disorders. American Journal of Psychiatry, 149, 936-943.

Kabat-Zinn, J., Wheeler, E., Light, T., Skillings, A., Scharf, M., Cropley, T., Hosmer, D. \& Bernhardt, J. (1998). Influence of a mindfulness meditation-based stress reduction intervention on rates of skin clearing in patients with moderate to severe psoriasis undergoing phototherapy (UVB) and photochemotherapy (PUVA). Psychosomatic Medicine, 60 , 625-632.

Kaplan, K., Goldenberg, D. \& Galvin-Nadeau, M. (1993). The impact of a meditation-based stress reduction program on fibromyalgia. General Hospital Psychiatry, 15, 284-289.

Kiecolt-Glaser, J. K., McGuire, L., Robles, T. F., \& Glaser, R. (2002 a). Psychoneuroimmunology and psychosomatic medicine: Back to the future. Psychosomatic Medicine, 64, 15-28.

Kiecolt-Glaser, J. K., McGuire, L., Robles, T. F., \& Glaser, R. (2002 b). Psychoneuroimmunology: Psychological influences on immune function and health. Journal of Consulting and Clinical Psychology, 3, 537-547.

Kiecolt-Glaser, J., Robles, T., Heffner, K., Loving, T. \& Glaser, R. (2002). Psycho-oncology and cancer: Psychoneuroimmunology and cancer. European Society for Medical Oncology, 165-169.

Levy, S., Herberman, R., Lippman, M., D’Angelo, T. \& Lee, J. (1991). Immunological and psychosocial predictors of disease recurrence en patients with early-stage breast cancer. Behavioral Medicine, 17, 67-75.

Linehan, M. M. (1993a). Cognitive-behavioral treatment of borderline personality disorder. New York: Guilford Press.

Linehan, M. M. (1993b). Skills training manual for treating borderline personality disorder. New York: Guilford Press.

McIntyre, T. M., Fernandes, S. R. \& Pereira, M. G. (2002). Intervenção psicossocial breve na situação oncológica em contexto de grupo. In M. R. Dias \& Durá, E. (Coords), Territórios da psicologia oncológica (pp. 281-302). Lisboa: Climepsi.

Martín, A., \& García de la Banda, G. (2007). Las ventajas de estar presente: desarrollando una conciencia plena para reducir el malestar psicológico. International Journal of Clinical and Health Psychology, 7, 369-384.

Miller, J. J., Fletcher, K. \& Kabat-Zinn, J. (1995). Three-year follow-up and clinical implications of a mindfulness meditation-based stress reduction intervention in the treatment of anxiety disorders. General Hospital Psychiatry, 17, 192-200.

Monedo, M. \& Alonso C. (2003). Aspectos psicoemocionales. In V. Valentín (Ed.), Oncologia en Atención Primaria (pp. 665-680). Madrid: Nova Sidónia. 
Nezu, A., Nezu, C., Friedman, S., Faddis, S. \& Houts, P. (1998). Helping cancer patients cope: A problem-solving approach. Washington: American Psychological Association.

Olendzki, A. (2005). The roots of mindfulness. In C. K. Germer, R. D. Siegel \& P. R. Fulton (Eds.), Mindfulness and psychotherapy (pp. 241-261). New York: Guilford.

Ott, M. J., Norris, R. L. \& Bauer-Wu, S. M. (2006). Mindfulness meditation for oncology patients: A discussion and critical review. Integrative Cancer Therapies, 5, 98-108.

Pereira, M. G. \& Lopes, C. (2002). O doente oncológico e a sua família. Lisboa: Climepsi.

Reibel, D.K., Greeson, J.M., Brainard, G.C., \& Rosenzweig, S. (2001). Mindfulness based stress reduction and healthrelated quality of life in a heterogeneous patient population. General Hospital Psychiatry, 23, 183-192.

Rosenbaum, E. (2005). Here for now: Living well with cancer through mindfulness. Massachusetts: Satya House Publications.

Ryu, H., Jun, C., Lee, B., Choi, B., Kim, H. \& Chung, H. (1995). Effect of the qigong training on proportions of T lymphocyte subsets in human peripheral blood. American Journal of Chinese Medicine, 23, 27-36.

Schneider, R., Staggers, F., Alexander, C., Sheppard, W., Rainforth, M., Kondwani, K., Smith, S. \& King, C. (1995). A randomised controlled trial of stress reduction for hypertension in older African Americans. Hypertension, 26, 820-827.

Segal, Z., Williams, J. M. \& Teasdale, J. D. (2002). Mindfulness-based cognitive therapy for depression: A new approach to preventing relapse. London: The Guilford Press.

Shapiro, S L., Bootzin, R. R., Figueiredo, A. J., Lopez, A. M. \& Schwartz, G. E. (2003). The efficacy of mindfulness-based stress reduction in the treatment of sleep disturbance in women with breast cancer: An exploratory study. Journal of Psychosomatic Research, 54, 85-91.

Singh, B., Berman, B., Hadhazy, V. \& Creamer, P. (1998). A pilot study of cognitive behaviour therapy in fibromyalgia. Alternative Therapy Health Medicine, 4, 67-70.

Solberg, E., Halvorsen, R., Sundgot-Borgen, J., Ingjer, F. \& Holen, A. (1995). Meditation: A modulator of the immune response to physical stress? A brief report. British Journal of Sports Medicine, 29, 255-257.

Speca, M., Carlson, L., Goodey, E., \& Angen, M. (2000). A randomized, wait-list controlled clinical trial: The effect of a mindfulness meditation-based stress reduction program on mood and symptoms of stress in cancer outpatients. Psychosomatic Medicine, 62, 613-622.

Speca, M., Carlson, L., MacKenzie, M., \& Angen, M. (2006). Mindfulness-based stress reduction (MBSR) as an intervention for cancer patients. In R. A. Baer (Ed.), Mindfulness-based treatment approaches: Clinician's guide to evidence base and applications (pp. 239261). London: Academic Press.

Spiegel, D. (1995). How do you feel about cancer now? - Survival and Psychosocial Support. Public Health Report, 110, 298-300.

Spiegel, D. (1996). Cancer and depression. British Journal of Psychiatry Suppl., 109-116.

Spiegel, D., Sephton, S., Terr, A. \& Sittes, D. (1998). Effects of psychosocial treatment in prolonging cancer survival may be mediated by neuroimmune pathways. Annals of the New York Academy of Sciences, 840, 674-683.

Stewart, D., Cheung, A., Duff, S., Wong, F., McQuestion, A., Cheng, T., Purdy, L., \& Bunston, T. (2001). Attributions of cause and recurrence in long-term breast cancer survivors. Psychooncology, 10, 179-183.

Strain, J. J. (1998). Adjustment disorders. In J. F. Holland (Ed.), Psycho-oncology (pp. 509-517). New York: Oxford University Press. 
Tacón, A., Caldera, Y. \& Ronaghan, C. (2004). Mindfulness-based stress reduction in women with breast cancer. Families, Systems, \& Health, 22, 193-203.

Tacón, A., Caldera, Y. \& Ronaghan, C. (2005). Mindfulness, psychosocial factors, and breast cancer. Journal of Cancer Pain \& Symptom Palliation, 1, 45-53.

Taylor, D. N. (1995). Effects of a behavioural stress-management program on anxiety, mood, self esteem, and T-cell count in HIV positive men. Psychological Reports, 76, 451-457.

Trijsburg, R. W., Van Knippenberg, F. C. \& Rijpma, S. E. (1992). Effects of psychological treatment on cancer patients: A critical review. Psychosomatic Medicine, 54, 489-517.

Truant, T., \& Bottorff, J.L. (1999). Decision making related to complementary therapies: A process of regaining control. Patient Education and Counseling, 38, 131-142.

Twycross, R. (1999). Cuidados Paliativos. Lisboa: Climepsi.

Verhoef, M.J., Hilsden, R.J., \& O’Beirne, M. (1999). Complementary therapies and cancer care: An overview. Patient Education and Counseling, 38, 93-100.

Weiger, W., Smith, M., Boon, H., Richardson, M., Kaptchuk, T. \& Eisenberg, D. (2002). Advising patients who seek complementary and alternative medical therapies for cancer. Annals of Internal Medicine, 137, 889-903.

Zabora, J., Blanchard, C., Smith, E., Roberts, C., Glajchen, M., Sharp, J., Brintzenhofe-Szoc, K., Locher, J., Carr, E., Best-Castner, S., Smith, P., Dozier-Hall, D., Polinsky, M. \& Hedlund, S. (1997). Prevalence of psychological distress among cancer patients across the disease continuum. Journal of Psychosocial Oncology, 15, 73-86.

Zabora, J., BrintzenhofeSzoc, K., Curbow, B., Hooker, C., \& Piantadosi, S. (2001). The prevalence of psychological distress by cancer site. Psycho-Oncology, 10, 19-28.

\section{Health promotion in oncologic disease: Stress reduction intervention based on mindfulness}

The main goal of this revision article is (1) to convey a general vision and discussion about mindfulness meditations and its clinical practical value in oncology and (2) to report, in detail - assessing critically the existing research data, as well as the emergent one about mindfulness meditation - an intervention to people with cancer disease. In addition, it wishes to bring up the hypothesis of a program of health promotion and stress reduction, based on mindfulness, exploring its effects in terms of biologic and psychosocial variables. Foreign studies refer significant levels of change on psychosocial items of assessment, in the different moments of assessment. In that way, the intervention has relative theoretical support, even though this theoretical basis needs empirical refinement. It's hoped that the eventual application of the intervention here described will allow to work the factors taken as beneficial to promote a better adjustment to the oncologic disease, rising proactively the actions of the individual has an informed intervenient of its process of progressive adaptation to the disease.

KEYWORDS: Mindfulness; Oncology; Stress; Intervention; Theoretical study. 